\title{
AUGMENTED INTEGRATED FUZZY NEURAL NETWORK LEARNING MODEL IN STRUCTURAL ENGINEERING
}

\author{
Shih-Lin Hung and Jan, C.C. \\ Department of Civil Engineering, National Chiao Tung University \\ 1001 Ta Hsueh Road, Hsinchu, Taiwan 300, R.O.C.
}

\begin{abstract}
Solving engineering problems is a creative, experiential process. An experienced engineer generally solves a new problem by recalling some similar instances examined before and applying what he learned from the present problem, through adaptation or synthesis. According to such a method, the IFN learning model was developed and implemented as a computational model for problem solving. This model has been applied to design problems involving a complicated steel structure. Computational results indicate that the IFN model can learn the complicated problems within a reasonable computational time owing to its simplicity. The learning performance of IFN, however, relies heavily on the values of some working parameters selected on a trial and error basis. In this work, we present an augmented IFN learning model by integrating a conventional IFN learning model with two novel approaches: a correlation analysis in statistics and a self-adjustment in mathematical optimization - to facilitate the search for appropriate working parameters in the conventional IFN. The problem of arbitrary trial and error selection of the working parameters is avoided in the augmented IFN learning model.
\end{abstract}

Keywords:Artificial Neural Networks, Supervised Learning Models, Unsupervised Fuzzy Learning Model, Structural Design.

\section{TNTRODUCTION}

Solving engineering problems, such as analysis and design problems, is a creative, experiential process in which the experiences and combined knowledge of engineers serve as resources. An experienced engineer generally solves a new problem in the following stages. First, he or she recalls some similar instances that have been solved before while properly considering the functional requirements of those instances. Then, the engineer attempts to derive the solution from these similar instances through adaptation or synthesis. After the problem is solved, the new instance is then stored in her/his memory as an additional knowledge resource for solving further problems in the future.

The above stages can be implemented as a computational model for problem solving that utilizes a case base of previously solved problems when solving a new problem. In symbolic artificial intelligence (AI), case-based reasoning [1] is an effective means of facilitating computer program development that attempts to solve problems by directly accessing the case base. Artificial neural networks (ANNs), on the other hand, constitute a different $\mathrm{AI}$ approach that has made rapid advances in recent years. Such networks have the ability to develop, from training instances, their own solutions to a class of problems [2-5].

Recently, authors [6][7] presented an integrated fuzzy neural network (IFN) learning model in structural engineering. The IFN learning model combined a novel unsupervised fuzzy neural network (UFN) reasoning model with a supervised neural network learning model using the adaptive L-BFGS learning algorithm [8]. The IFN learning model was applied to steel beam design problems. That work contended that the IFN learning model is a robust and effective ANN learning model. In addition, the IFN model can interpret a large number of instances for a complicated engineering problem within a reasonable computational time owing to its simplicity in computation. However, the performance of the IFN learning model is heavily affected by some working parameters that are problem-dependent and obtained via trial and error.

Herein, we present a more effective neural network learning model, called the augmented IFN learning model, by integrating a conventional IFN learning model with two newly developed approaches: a correlation analysis in statistics and self-adjustment in mathematical optimization. The first approach, 
correlation analysis in statistics, is employed to assist users in determining the appropriate working parameter to be used in the fuzzy membership function. The second approach, self-adjustment in mathematical optimization, is used to obtain appropriate weights, systematically, for each decision variable required in the input of training instances. The augmented IFN learning model proposed herein is applied to the preliminary design of steel structure buildings.

\section{REVIEW OF IFN LEARNING MODEL}

The IFN learning model combines two sub-ANN learning models [6]. One is a novel unsupervised fuzzy neural network (UFN) reasoning model: a singlelayered laterally-connected network with an unsupervised competing learning algorithm. The other is an offline assistant model: a supervised neural network learning model with the adaptive L-BFGS learning algorithm [8]. The IFN learning model is schematically depicted in Fig. 1.

The learning stage in the IFN model is performed in two sub-ANN models concurrently. First, the offline assistant supervised neural network model is trained, based on the adaptive L-BFGS supervised learning algorithm. In the UFN reasoning model, however, the learning process simply involves selecting appropriate working parameters for the fuzzy membership function and weights for each decision variable in the input.

After learning in the UFN and in the assistant supervised learning model is completed, any new instance $X$ can be solved via the IFN learning model. The reasoning in UFN is performed through a singlelayered laterally-connected network with an unsupervised competing algorithm and it is implemented in three steps. The first step involves searching for some instances from the instance base that resemble the new instance $X$ according to their inputs.

$d_{i j}=\operatorname{diff}\left(U_{i, i}, U_{j, i}\right)=\sum_{m=1}^{M} \alpha_{m}\left(w_{i} u_{i}^{m}-w_{j} u_{j}^{m}\right)^{2}$

The second step entails representing the relationships among the new instance and its similar instances, with $d_{X j}$ less than $R_{\max }$.

$\mu\left(d_{X j}\right)=\left\{\begin{array}{lll}0 & \text { if } & d_{i j} \geq R_{\max } \\ \frac{R_{\max } R_{\min }-R_{\min } d_{i j}}{\left(R_{\max }-R_{\min }\right) d_{i j}} & \text { if } \quad R_{\min }<d_{i j}<R_{\max } \\ 1 & \text { if } d_{i j} \leq R_{\min }\end{array}\right.$

The final step involves generating the output $X_{o}$ vector of instance $X$ by synthesizing the outputs of its similar instances according to their associated fuzzy membership values using center of gravity (COG) or mean of maximum (MOM) methods [6][7].

The reasoning process of the UFN depends on determining the degree of similarity among $X$ and $U_{j}$. Consequently, no solution can be generated by the
UFN reasoning model, if the new instance entirely differs from all instances in the instance base, e.g. all $d_{X i}$ are greater than $R_{\max }$. In addition, using inappropriate working parameters would allow for the possibility that no similar instances can be derived. For the above issues, the under-trained adaptive LBFGS supervised neural network is used as an assistant system to generate an approximate output for the new instance.

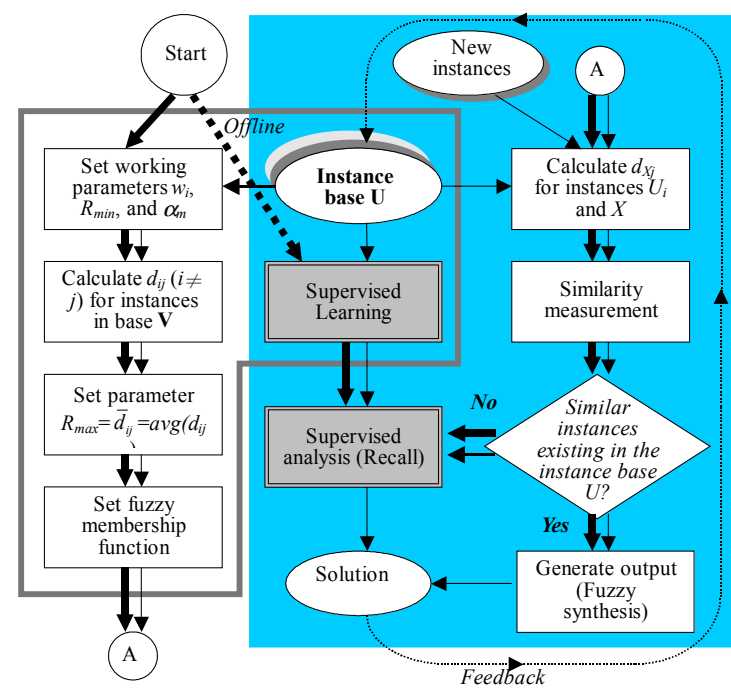

$\begin{aligned} & \text { Learning stage in the IFN learning model (including UFN } \\ & \text { reasoning model and supervised learning model) } \\ & \text { Verification stage in the IFN learning model (including UFN } \\ & \text { reasoning model and supervisedlearning model) }\end{aligned}$
Procedure of UFN reasoning model
$\quad$ Procedure of the assistant supervised learning model

Figure 1 Conventional IFN learning model.

\section{AUGMENTED IFN LEARNING MODEL}

In the conventional IFN learning model, working parameters, such as $w_{j}, \alpha_{m}, R_{\max }$, and $R_{\min }$, are selected subjectively by users and, generally, on a trial and error basis. Consequently, the learning performance is highly affected by these parameters, especially $R_{\max }$ and $\alpha_{m}$. In this work, two novel approaches are employed for assisting the users to determine these parameters and weights systematically.

\subsection{Correlation Analysis for $R_{\max }$ in Fuzzy Membership Function}

In conventional IFU, the similarity measurement between two instances heavily depends on the value of parameter $R_{\max }$. A small value of $R_{\max }$ implies that a 
strict similar relationship between instances is utilized. Consequently, most of the instances are sorted as dissimilar. Consequently, few similar instances to the new instance can be found and, ordinarily, no solution can be generated via the UFN reasoning model. On the other hand, a loose similar relationship is adopted under the case of a larger $R_{\max }$. Accordingly, a large number of instances are taken to be "similar instances" and the solution generated via these similar instances, is inferior. Here, the linear correlation analysis in statistics is employed to facilitate the determination of appropriate value of $R_{\max }$ in the fuzzy membership function. The analysis is a process which aims to measure the strength of the association between two sets of variables that are assumed to be linearly related.

For the above instance base $\mathbf{U}$ with $N$ instances, the correlation analysis in the fuzzy membership function is implemented in the following steps. The first step is to determine the degree of difference between any two instances in the base $\mathbf{U}$ using the aforementioned function of degree difference in Eq.(1). Hence, a total of $\left(C_{2}^{N}+N\right)$ resembling samples, $S_{i j}\left(U_{i, o}, U_{j, o}, d_{i j}\right)$, can be compiled. A resembling sample contains two instances' outputs $\left(U_{i, o}\right.$ and $\left.U_{j, o}\right)$ and the corresponding degree of difference $\left(d_{i j}\right)$. Thereafter, two arrays, $A_{t}$ and $B_{t}$, can be assorted from resembling samples in the case of $d_{i j}$ less than or equal to a prescribed value, say $t$. The elements in $A_{t}$ and $B_{t}$ are the first and second items, respectively, of these resembling samples. Next, the accumulative correlation coefficient, Ac_CORREL $\left(A_{t}, B_{t}, t\right)$, is calculated for arrays $A_{t}$ and $B_{t}$ with the degree of difference less than or equal to $t$. Assume that for a total $P$ resembling samples with $d_{i j}$ less than a prescribed $t$, the arrays $A_{t}$ and $B_{t}$ can be denoted as

$A_{t}=\left\{a_{k} \mid a_{k}=U_{i, o} \in S_{i j}\right.$, for $\left.d_{i j} \leq t\right\}=\left\{a_{1}, a_{2}, \ldots, a_{p}\right.$ $B_{t}=\left\{b_{k} \mid b_{k}=U_{j, o} \in S_{i j}\right.$, for $\left.d_{i j} \leq t\right\}=\left\{b_{1}, b_{2}, \ldots, b_{p}\right\}$ The value of the accumulative correlation coefficient equals

$$
\begin{aligned}
& \operatorname{Ac} \_\operatorname{CORREL}\left(A_{t}, B_{t}, t\right)=\frac{\operatorname{Cov}\left(A_{t}, B_{t}, t\right)}{\sigma_{A_{t}} \sigma_{B_{t}}} \\
& \operatorname{Cov}\left(A_{t}, B_{t}, t\right)=\frac{1}{p} \sum_{k=1}^{p}\left(a_{k}-\mu_{A_{t}}\right)\left(b_{k}-\mu_{B_{t}}\right) \quad \text { s.t. } d_{i j} \leq t
\end{aligned}
$$

where $\sigma_{A_{t}}$ and $\sigma_{B_{t}}$ are standard errors of arrays $A_{t}$ and $B_{t} ; \mu_{A_{t}}$ and $\mu_{B_{t}}$ are the means of $A_{t}$ and $B_{t}$. The formulas expressed in (3) and (4) represent the relationship between the accumulative correlation coefficient to any value of $t$. An accumulative correlation curve can be plotted as a function of $t$ and $A c \_C O R R E L\left(A_{t}, B_{t}, t\right)$. Note that the appropriate $R_{\max }$ equals a certain value of $t$ such that instances in the instance base $\mathbf{U}$ have a certain degree of correlation.
Obviously, the smaller the $t$ implies a larger accumulative correlation coefficient, indicating a strong relationship between the two arrays, e.g., the strongest correlation, $t=0$, between the two arrays refers to the case in which the instances in the two sets are identical and the value of $A c_{-} \operatorname{CORREL}\left(A_{t}, B_{t}, t\right)$ equals one. In such a case, no solution to a new instance can be generated via the UFN reasoning model except for when identical instances exist in the instance base. In order to avoid this issue here, we set Ac_CORREL $\left(A_{t}, B_{t}, t\right)$ equal to 0.8 as the lower bound for similarity measurement. The value of $t$ corresponding to this lower bound is adopted as the appropriate value of $R_{\max }$.

\subsection{Self-Adjustment for Selecting Weights $\alpha_{m}$}

Except for $R_{\max }$, the selected weights $\alpha_{m}$ also significantly affect the learning performance for the conventional IFN. This occurrence has been investigated in the earlier work [7]. The learning results indicated that significant improvements were achieved as the weights were gradually updated via a basis of heuristic knowledge associated with learning problems. In this study, a more systematical approach self-adjustment based on mathematical optimization is adopted to facilitate the search for appropriate weights.

For the above instance base $\mathbf{U}$ with $N$ instances, the self-adjustment approach can be briefly stated as consisting of the following steps. First, set up the corresponding working parameters, $R_{\max }, R_{\min }$, and $w_{j}$. where $R_{\max }$ is determined using the aforementioned correlation analysis approach; and where parameter $R_{\min }$ and weights $w_{j}$, are set as constants in this work. Meanwhile, weights $\alpha_{m}$ for each decision variable in the input are directly initialized as one. Then, based on these working parameters, the outputs for training instances are found via the UFN reasoning model. Then the error, $E_{i}$, between the computed and desired outputs, $Y$ and $U_{i, o}$, for training instance $U_{i}$, is calculated. The system error, $E$, for a total $N$ instances is then defined as half of the average sum of errors and denoted as

$$
\begin{aligned}
& E=\frac{1}{2 N} \sum_{i=1}^{N} E_{i} \\
& E_{i}=\sum_{k=1}^{K}\left(y^{k}-o_{i}^{k}\right)^{2}
\end{aligned}
$$

where $o_{i}^{k}$ and $y^{k}$ are the $k$ th items of data in desired and computed outputs, $U_{i}$ and $Y$, respectively. Note that the system error is an implicit function of the weights $\alpha_{m}$ as $E\left(\alpha_{m}\right)$.

Weights $\alpha_{m}$ in the UFN reasoning model are adjusted to reduce the system error as much as possible. This goal can be achieved if a set of appropriate weights, $\alpha_{m}$, are used. The problem, then, can then be considered as an unconstrained optimization problem; that is searching a set of 
optimum weights by iteration to minimize the system error. In the mathematical optimization approaches, the conjugate gradient (CG) method has been proved an efficient means of solving the problem. The weights $\alpha_{m}$ are updated in each iteration, say the $(s+1) t h$ iteration, as $\alpha_{m}^{(s+1)}=\alpha_{m}^{(s)}+\lambda \mathbf{d}^{(s)}$. The term $\square$ is step length and is set as a constant in this work. The search direction $\mathbf{d}^{(s)}$ is defined as

$\begin{cases}\mathbf{d}^{(s)}=\mathbf{g}^{(s)}=-\nabla E\left(\alpha_{m}^{(s)}\right) & \text { if } s=0 \\ \mathbf{d}^{(s)}=\mathbf{g}^{(s)}+\beta^{(s)} \mathbf{d}^{(s-1)} & \text { if } s>0\end{cases}$

where $\beta^{(s)}=\frac{\left(\mathbf{g}^{(s)}\right)^{T} \mathbf{g}^{(s)}}{\left(\mathbf{g}^{(s-1)}\right)^{T} \mathbf{g}^{(s-1)}}$, and $\mathbf{g}^{(s)}=-\nabla E\left(\alpha_{m}^{(s)}\right)$

The iteration is terminated as the value of $\left\|\mathbf{g}^{(s+1)}\right\|$ or $\left|E\left(\alpha_{m}^{(s+1)}\right)-E\left(\alpha_{m}^{(s)}\right)\right|$ is sufficiently small. The term $\mathbf{g}^{(s)}$ is the negative gradient vector of function $E\left(\alpha_{m}\right)$. For simplicity, the superscript $(s)$, denoted as the $s$ th iteration, is ignored. Hereinafter, vector $\mathbf{g}$ can be derived as

$$
\begin{aligned}
& \mathbf{g}=\frac{\partial E\left(\alpha_{m}\right)}{\partial \alpha_{m}}=\frac{1}{2 N} \sum_{i=1}^{N} \frac{\partial E_{i}}{\partial \alpha_{m}} \\
& \frac{\partial E_{i}}{\partial \alpha_{m}}=2 \sum_{k}\left(y^{k}-o_{i}^{k}\right)\left(\sum_{p} f\left(d_{i p}\right)\right)^{-2} x \\
& \quad\left(\sum_{p} o_{p}^{k} f^{\prime}\left(d_{i p}\right)\left(u_{i}^{m}-u_{p}^{m}\right)^{2} \sum_{p} f\left(d_{i p}\right)\right. \\
& \left.\quad-\sum_{p} f\left(d_{i p}\right) o_{p}^{k} \sum_{p} f^{\prime}\left(d_{i p}\right)\left(u_{i}^{m}-u_{p}^{m}\right)^{2}\right)
\end{aligned}
$$

\subsection{Augmented IFN Learning Model}

Figure 2 schematically depicts the procedures of the augmented IFN learning model. Instead of using a constant working parameter $R_{\max }$ as in conventional IFN, the appropriate parameter is determined using correlation analysis. Meanwhile, the appropriate weights $\alpha_{m}$ are adapted during the self-adjustment process through a mathematical approach. The procedure of the augmented IFN learning model can be summarized as follows:

Self-organized learning phase:

Step 0: Train the adaptive supervised L-BFGS learning neural network model offline.

Step 1: Initialize parameter $R_{\max }$ as constant $10^{-5}$ and weight $\alpha_{m}$ for each decision variable on a heuristic basis or by trial and error.

Step 2: Calculate the degree of difference $d_{i j}(i \neq j)$ among all instances in base $\mathbf{V}$.

Step 3: Determine the parameter $R_{\max }$ using correlation analysis and set $R_{\max }=t$ such that $A c \_C O R R E L\left(A_{t}, B_{t}, t\right)=0.8$.

Step 4: Set the fuzzy membership function, $\mu\left(d_{i j}\right)=f\left(R_{\min }, R_{\max }, d_{i j}\right)$, defined in Eq.(2).
Step 5: Adjust the weight $\alpha_{m}$ for each decision variable in input using the self-adjustment approach.

Analysis phase (After the learning phase is completed):

Step 6: Present the new (unsolved) instance $X$ to the UFN reasoning model and perform a similarity measurement between $X$ and instance $U_{i}$ in the base $\mathbf{U}$ using a singlelayered lateral-connected competing network.

Step 7: If more than one similar instance is found in Step 6, generate the solution $X_{o}$ for the new instance using fuzzy synthesis approaches in Eq. (4) or (5) and go to Step 9. Otherwise, go to next step.

Step 8: Compute the solutions via the under-trained assistant adaptive L-BFGS supervised learning model and go to next step.

Step 9: Feedback the new instance into the base $\mathbf{U}$. Meanwhile, further learning in the assistant supervised learning model is launched offline.

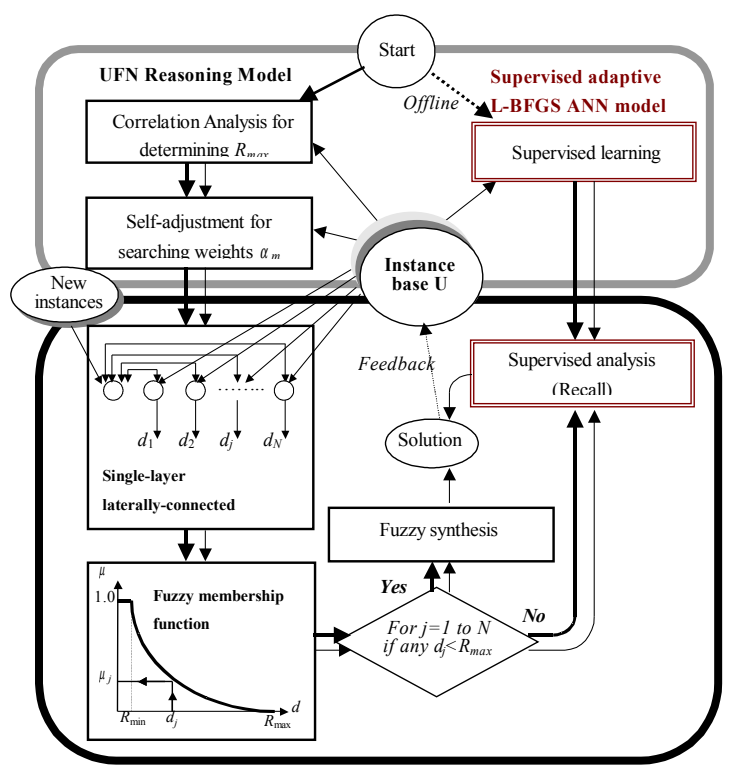

Figure 2 Augmented IFN learning model.

\section{APPLICATIONS}

In this work, the augmented IFN learning model is trained to learn how to implement the preliminary design of buildings satisfying the conditions of utility, safety, and economy in only one design cycle. In the complete design of a structure, the preliminary design stage is mainly a creative, experiential process that involves the choice of structure type, selection of the material, and determination of the sections of beams and columns in the structure. An experienced engineer is likely to carry out this stage more quickly than does an inexperienced one. The basic configuration of the structure at this stage should satisfy the specified, such as LRFD [9] for steel structures. To satisfy the prescribed constrains and achieve minimum 
expenditure for materials and construction, this stage becomes a looped optimization decision-making process. Hence, a good initial development of the basic form, with sections of beams and columns satisfying the aforementioned constraints, will reduce the number of redesign cycles. The present example involves a complete design structure which satisfies the conditions that the service loads should not exceed the strength of the members; the drifts should be within the prescribed limits, and the structure should be economical in material (e.g. minimum weight), construction, and overall cost. For simplicity, only regular buildings with a rectangular layout, such as most factory buildings, are considered herein. Also, the beams in every floor have the same sectional dimensions, as do the columns.

416 instances are used in this example. They are randomly divided into 380 training instances and 36 verification instances. Seven decision variables are used as inputs to determine the sections of beams and columns of a building that satisfies the given specifications. The seven decision variables and their limits are described as following:

$$
\begin{aligned}
& \text { 1. Number of stories }=[9, \quad 10 \text {, } \\
& 11,12,13,14,15] \text {. }
\end{aligned}
$$

6. Live load $\left(\mathrm{kgw} / \mathrm{m}^{2}\right)=200$ to 350 .

7. Wall load $(\mathrm{kgw} / \mathrm{m})=[100,200]$.

Other corresponding decision variables used in the stage of preliminary design are assumed to be constant. Here, a building with three groups of steel elements in both beams and columns is considered. The three groups are upper, medium, and lower. An instance contains seven decision variable inputs and six data items as outputs.

The parameters $R_{\min }$ and $w_{i}$ are set as constant $10^{-5}$ and one, respectively. The weights $\alpha_{m}$, however, are initialized as $[1,1,1,1,1,1,1]$ for the seven decision variables. Using these parameters and weights, correlation analysis in the augmented IFN learning model is performed first to determine the working parameter $R_{\max }$. With the value of Ac_CORREL $\left(A_{t}, B_{t}, t\right)$ equal to 0.8 , the values of $t\left(=R_{\max }\right)$ are obtained. They are 0.12 for beams and 0.178 for columns, respectively.

After the fuzzy membership function is defined, the self-adjustment approach is then launched to obtain the adequate weights $\alpha_{m}$ for each decision variable in the input. The weights $\alpha_{m}$ for each decision variable are updated from $[1,1,1,1,1,1,1]$ to $[1.471,1.369$,
$0.416,0.008,1.104,0.825,0.513]$ for beams and to $[1.106,0.997,0.542,0.009,1.232,0.999,0.997]$ for columns. Interestingly, the weights of the fourth decision variable in beams and columns are both selfadjusted close to zero. This observation indicates that this decision variable (number of bays in both directions) is insignificant in the input. Consequently, this decision variable can be neglected. The 36 verification instances are used to verify the learning performance of the augmented and conventional IFN learning models, respectively. Notably, the augmented IFN is verified on the basis of the newly adjusted weights $\alpha_{m}$ with the adequate $R_{\max }$. The working parameters and weights used in the conventional IFN, however, are selected on a trial and error basis. Table 1 summarizes the learning results for thirty-six verification instances. According to this table, the average percentage errors for beams and columns are 13.81 and 9.36 for the conventional IFN learning model. However, these errors are reduced to 6.17 and 6.1 for beams and columns, respectively, for the augmented IFN learning model. The augmented IFN learning model significantly improves in terms of learning.

TABLE 1. Results of Preliminary Structural Design Problem

\begin{tabular}{c|c|c|c|c}
\hline \hline \multirow{4}{*}{ Error (\%) } & \multicolumn{4}{|c}{$\begin{array}{c}\text { Number of items in output for } \\
\text { verification instances }\end{array}$} \\
\cline { 2 - 5 } & $\begin{array}{c}\text { Conventional IFN } \\
\text { Learning Model }\end{array}$ & $\begin{array}{c}\text { Augmented IFN } \\
\text { Learning Model }\end{array}$ \\
\cline { 2 - 5 } & Beam & Column & Beam & Column \\
\hline $0 \sim 5$ & 12 & 43 & 52 & 51 \\
$5 \sim 10$ & 29 & 29 & 39 & 31 \\
$10 \sim 15$ & 21 & 22 & 13 & 20 \\
$15 \sim 20$ & 29 & 10 & 3 & 6 \\
$>20$ & 17 & 4 & 1 & 0 \\
\hline Average & $13.81 \%$ & $9.36 \%$ & $6.10 \%$ & $6.17 \%$ \\
Error & & & & \\
\hline
\end{tabular}

Note: Each instance has six items, three beams and columns, in output.

\section{CONCLUUSIONS}

This work presents an augmented IFN learning model by integrating two newly developed approaches into a conventional IFN learning model. These approached are a correlation analysis in statistics and self-adjustment in mathematical optimization, which collaboratively enhance the learning capability of the conventional IFN. The augmented IFN learning model proposed herein is applied to engineering design problem of preliminary design of stell structures. Based on the results of this work, we can conclude the following:

1. The problem of arbitrary trial and error selection of the working parameter $\left(R_{\max }\right)$, in fuzzy 
membership function, encountered in the conventional IFN learning model is avoided in the newly developed augmented IFN learning model. Thus, the new learning model provides a more solid systemic foundation for IFN learning than the conventional IFN learning model.

2. In the conventional IFN learning model, the weights $\alpha_{m}$, denoting the importance of the $m$ th decision variable in the input, are set on a trial and error basis. This problem is avoided by the newly developed learning model. Instead of an assumed constant, the appropriate weights are determined through the self-adjustment approach in mathematical optimization. Therefore, the augmented IFN learning model provides a more solid mathematical foundation for neural network learning.

3. For each training instance, decision variables in the input are generally selected subjectively by users. As a result, some trivial decision variables may be adopted in the input for some complicated examples. Based on the self-adjustment approach, the importance of a decision variable in an input can be derived systematically. Therefore, insignificant or redundant decision variables in the input can be neglected.

\section{Acknowledgments}

The authors would like to thank the National Science Council of the Republic of China for financially supporting this research under Contract No. NSC 86-2221-E-009-070.

\section{REFERENCES}

[1] Carbonell, J. G. (1981). "A computional model of analogical problem solving." Proceedings of the Seventh International Joint Conference on Artificial Intelligence 1: 147-152.

[2] Adeli, H., and Hung, S. L. (1995). Machine Learning -- Neural Networks, Genetic Algorithms, and Fuzzy Systems. John Wiley \& Sons, New York, N.Y.

[3] Ghaboussi, J., Garrett, J. H., and Wu, X. (1991). "Knowledge-based modeling of material behavior and neural networks." J. Engrg. Mech., 117(1), 132-153.

[4] Gunaratnam, D. J., and Gero, J. S. (1994). "Effect of representation on the performance of neural networks in structural engineering applications." Microcomputers in Civ. Engrg., 9(2), 97-108.

[5] Hajela, P. and Berke, L. (1991). "Neurobiological computational modes in structural analysis and design." Computers and Struct., 41(4), 657-667.
[6] Hung, S.L. and Jan, J.C. (1997), "Machine learning in engineering design- An unsupervised fuzzy neural network learning model," Proceedings of Intelligent Information Systems (IIS'97), Grand Bahama, December 8-10, IEEE Computer Society, California, pp.156-160.

[7] Hung, S.L. and Jan, J.C. (1999), "Machine learning in engineering analysis and design- An integrated fuzzy neural network learning model," ComputerAided Civ and Infrastructure Engrg., 14(3), 207-219.

[8] Hung, S. L., and Lin, Y. L. (1994). “Application of an L-BFGS neural network learning algorithm in engineering analysis and design." Proceedings of The Second National Conference on Structural Engineering- Vol. III, Nov. 4-6, Nantou, Taiwan, R.O.C., 221-230 (in Chinese).

[9] Manual of steel construction - load \& resistance factor design (1994). America Institute of Steel Construction, Inc., Chicago, IL. 